\title{
Encoding Difficulty Promotes Postlearning Changes in Sleep Spindle Activity during Napping
}

\author{
Christina Schmidt, ${ }^{1,2,3}$ Philippe Peigneux, ${ }^{2}$ Vincenzo Muto, ${ }^{4}$ Maja Schenkel, ${ }^{1}$ Vera Knoblauch, ${ }^{1}$ Mirjam Münch, ${ }^{1}$ \\ Dominique J.-F. de Quervain, ${ }^{5}$ Anna Wirz-Justice, ${ }^{1}$ and Christian Cajochen ${ }^{1}$ \\ ${ }^{1}$ Centre for Chronobiology, Psychiatric University Clinics, CH-4025 Basel, Switzerland, ${ }^{2}$ Cyclotron Research Centre and ${ }^{3}$ Department of Cognitive \\ Psychology, University of Liège, B-4000 Liège, Belgium, ${ }^{4}$ Department of Psychology, II University of Naples, 81100 Caserta, Italy, and ${ }^{5}$ Division of \\ Psychiatry Research, University of Zurich, CH-8057 Zurich, Switzerland
}

Learning-dependent increases in sleep spindle density have been reported during nocturnal sleep immediately after the learning session. Here, we investigated experience-dependent changes in daytime sleep EEG activity after declarative learning of unrelated word pairs. At weekly intervals, 13 young male volunteers spent three $24 \mathrm{~h}$ sessions in the laboratory under carefully controlled homeostatic and circadian conditions. At approximately midday, subjects performed either one of two word-pair learning tasks or a matched nonlearning control task, in a counterbalanced order. The two learning lists differed in the level of concreteness of the words used, resulting in an easier and a more difficult associative encoding condition, as confirmed by performance at immediate cued recall. Subjects were then allowed to sleep for $4 \mathrm{~h}$; afterward, delayed cued recall was tested. Compared with the control condition, sleep EEG spectral activity in the low spindle frequency range and the density of low-frequency sleep spindles $(11.25-13.75 \mathrm{~Hz})$ were both significantly increased in the left frontal cortex after the difficult but not after the easy encoding condition. Furthermore, we found positive correlations between these EEG changes during sleep and changes in memory performance between pre-nap and post-nap recall sessions. These results indicate that, like during nocturnal sleep, daytime sleep EEG oscillations including spindle activity are modified after declarative learning of word pairs. Furthermore, we demonstrate here that the nature of the learning material is a determinant factor for sleep-related alterations after declarative learning.

Key words: daytime sleep; sleep spindles; declarative memory; encoding difficulty; EEG; human

\section{Introduction}

Sleep is deemed to play a prominent role in the processing of recently acquired memory traces (Maquet, 2001; Stickgold and Walker, 2005; Walker and Stickgold, 2006). In humans, it has been proposed that specific sleep stages are involved in the consolidation of different memory domains (Smith, 2001; Rauchs et al., 2005). Evidence suggests that rapid eye movement sleep (REMS) is beneficial for procedural, nondeclarative memory (Smith, 1995; Plihal and Born, 1997), whereas non-REMS (NREMS) participates predominantly in the consolidation of declarative memories (Plihal and Born, 1997). In line with the idea that sleep affects memory consolidation, practice of memory tasks conversely affects the architecture of posttraining sleep (Meier-Koll et al., 1999; Maquet et al., 2000; Gais et al., 2002; Peigneux et al., 2003, 2004; Huber et al., 2004). In particular,

Received March 31, 2006; revised July 18, 2006; accepted July 18, 2006.

This work was supported by grants from the Swiss National Science Foundation, the Lundbeck-Belgian College of Neuropharmacology and Biological Psychiatry, the II University of Naples, and Eidgenössische Stipendienkommission für Ausländische Studierende. C.S. was supported by the Belgian National Foundation of Scientific Research P.P. was supported by the Interuniversity Attraction Pole P5/04. We thank Dr. Wim Martens for his helpful comments on the spindle detection algorithm; C. Renz, G. Balestrieri, and M.-F. Dattler for their help in data acquisition; and Drs. I. Willems and I. Amrhein for medical screenings.

Correspondence should be addressed to Christian Cajochen, Center for Chronobiology, Psychiatric University Clinics, Wilhelm-Klein Strasse 27, CH-4025 Basel, Switzerland. E-mail: christian.cajochen@upkbs.ch.

DOI:10.1523/JNEUROSCI.2464-06.2006

Copyright $\odot 2006$ Society for Neuroscience $\quad$ 0270-6474/06/268976-07\$15.00/0 sleep electroencephalographic (EEG) activity in the delta (0.5$4.5 \mathrm{~Hz})$ and spindle $(12-15 \mathrm{~Hz})$ range seems to be implicated in the practice of declarative memory tasks (Gais et al., 2002; Schabus et al., 2004, 2005; Clemens et al., 2005). Interestingly, sleep spindles are temporally related to the occurrence of hippocampal ripples (Siapas and Wilson, 1998; Sirota et al., 2003). Thus, their coactivation is believed to be important for NREMS-dependent processes of consolidation (Buzsaki, 1998), during which associative memories are gradually translated from hippocampal to neocortical stores (Chrobak and Buzsaki, 1996).

An important confounding factor in NREMS and declarative memory studies is that both EEG delta and spindle activity during sleep depend on the amount of previous wakefulness (homeostatic pressure) and the phase of endogenous circadian rhythms (Dijk and Czeisler, 1995; Knoblauch et al., 2003a,b). Furthermore, the intrinsic nature of the memory task might be a determinant factor in the occurrence of posttraining EEG oscillations during sleep. For instance, it was hypothesized that learning unrelated word pairs depends to a larger degree than related pairs on newly formed, hippocampally mediated associations (Stickgold, 2004). If sleep spindles and co-occurring hippocampal ripples are important for consolidation, then it can be hypothesized that memory tasks significantly challenging hippocampal activity would affect posttraining sleep EEG oscillations. We performed a declarative learning study in which sleep-wake-dependent (ho- 
Time of day (h)

\begin{tabular}{lllll}
\hline & & & 1 & \\
18 & 24 & 8 & 12 & 16
\end{tabular}

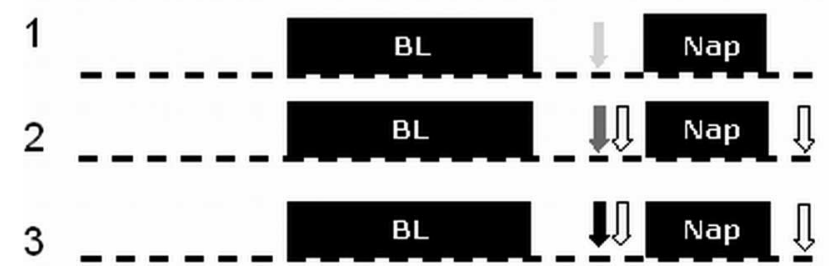

Figure 1. Overview of the protocol design for a subject with a habitual bedtime at midnight. Dashed lines indicate wakefulness under constant posture conditions, and horizontal black bars indicate scheduled sleep episodes. BL, Baseline night. Dark gray arrows, Difficult encoding condition; light gray arrows, easy encoding condition; white arrows, recall sessions; black arrow, control task.

meostatic) and circadian factors were carefully controlled for, whereas the degree of concreteness of the words constituting the lists of unrelated pairs to learn was manipulated. A manipulation at this level actually impacts on the associative encoding difficulty of the word pairs (Day and Bellezza, 1983). In a within-subject design, we investigated the repercussions of previous declarative learning on sleep architecture and sleep EEG oscillations, particularly spindle activity, during daytime sleep (i.e., napping) and whether the encoding difficulty of the material modulates this impact. Furthermore, we tested whether changes in posttraining sleep EEG oscillations correlate with post-napping learning performance.

\section{Materials and Methods}

Subjects. Thirteen young male volunteers (mean age of $24.4 \pm 1.8$ years; range of 21-28 years) gave their written informed consent for participating in this experiment approved by the local Ethical Committee. Exclusion criteria were reports of medical, psychiatric and sleep disorders, medication or drug consumption, alcohol abuse, excessive caffeine consumption or physical activity, shift work within the past 3 months, transmeridian travel or disturbances in the sleep-wake cycle within 1 month before the experiment, and extreme morning or evening chronotype [defined by scores $<12$ or $>23$ on the Torsvall-Åkerstedt morningevening types questionnaire (Torsvall and Åkerstedt, 1980)]. Drug-free status was controlled via an urinary toxicological analysis on admission to the study (Drug Screen Card Multi-6 for amphetamines, benzodiazepines, cocaine, methadone, opiates, and 9-tetrahydrocannabinol; von Minden, Moers, Germany). To assess the subject's rest-activity patterns, motor activity of the nondominant arm was recorded with actimeters (Cambridge Neurotechnologies, Cambridge, UK) the previous week and between the sessions along with sleep-wake logs.

Study protocol. An overview of the study design is illustrated in Figure 1. Each subject underwent three $24 \mathrm{~h}$ sessions in the chronobiology laboratory, separated by at least $7 \mathrm{~d}$. The precise schedule of each session was individually adapted according to the subject's habitual bedtime based on the average timing of the sleep midpoints, which were derived from the actimetry data during the preceding week. Subjects reported to the laboratory $7 \mathrm{~h}$ before habitual lights off on day 1 (Fig. 1). After the hook-up of the electrodes, subjects continuously stayed under constant posture conditions (semi-recumbent during scheduled wakefulness and fully recumbent during scheduled sleep), in dim light ( $<8$ lux during wakefulness, 0 lux during sleep episodes) and constant ambient temperature $\left(\sim 21^{\circ} \mathrm{C}\right)$. Subjective sleepiness was assessed every hour using visual analog scales (VAS) and the Karolinska Sleepiness Scale (KSS) (Åkerstedt and Gillberg, 1990). Hourly collected saliva samples were assayed for melatonin using a direct double-antibody radioimmunoassay validated by gas chromatography-mass spectroscopy with an analytical detectable dose of at least $0.65 \mathrm{pg} / \mathrm{ml}$ (Bühlmann Laboratories, Schönenbuch, Switzerland) (Weber et al., 1997). Polygraphic data (see below) were continuously recorded during each $24 \mathrm{~h}$ session. After lights off, subjects were allowed to sleep for $8 \mathrm{~h}$. Three hours after awakening, they performed one of the three experimental conditions, i.e., two different learning tasks and a control task in a counterbalanced order across the subjects. Two hours after the beginning of the experimental task [on average, at approximately midday (Fig. 1)], lights were turned off, and subjects were allowed to sleep for another $4 \mathrm{~h}$. Thirty minutes after lights on, taking into account the effect of sleep inertia on cognition (Jewett et al., 1999; HoferTinguely et al., 2005), delayed recall on the two learning tasks was assessed. Subjective cognitive strain, feeling of weariness, and boredom of the task were self-rated on five-point scales immediately after the end of each learning session (according to Gais et al., 2002).

Learning and nonlearning tasks. In each of the two learning tasks, subjects had to learn a randomized list of 154 unrelated word pairs displayed one by one on a computer screen using E-Prime software (Psychology Software Tools, Pittsburgh, PA). The list was presented twice (interpresentation interval of $2 \mathrm{~min}$; total duration of the encoding session, $\sim 50 \mathrm{~min}$ ). Each pair of words was displayed on the screen for $3 \mathrm{~s}$, followed by a white centered fixation cross for $5 \mathrm{~s}$, during which subjects were instructed to visually imagine a relationship between the two words of the pair in the aim to render mnemonic strategies more comparable across subjects (Gais et al., 2002; Schabus et al., 2004). After 5 s, the fixation cross changed from white to red (for $2 \mathrm{~s}$ ) to prepare the subject for the next word pair. Immediately after the end of the encoding session and $30 \mathrm{~min}$ after the end of the nap, recall performance for the word pairs was tested using a cued recall procedure: the first word of each pair was presented on the screen, while the subjects were asked to name the appropriate second word of the pair. There was no time limit for the recall. The subject's responses were written down on paper by the examiner. The order of presentation of the word pairs during the recall was pseudorandomized between subjects (four possible recall versions).

The nonlearning control task, designed according to Gais et al. (2002), served to mimic the learning task as much as possible but without the intentional learning component. One hundred fifty-four word pairs were presented using the same procedure as for the learning condition, but the subjects were instructed to count all letters containing curved lines on word pairs.

The words of the three lists were initially selected according to their length (words of 4-12 letters) based on a published database (Hager and Hasselhorn, 1994). Unrelated word pairs were used for all three conditions, i.e., pairs of words for which automatic preexisting associations were not available in memory. For instance, semantically highly related word pairs such as "cat-dog" or "river-ship" were never presented in any of the conditions. Emotionality and concreteness of the words were rated on a seven-point Likert scale in a pilot study $(n=12$; participants not included in the main experiment). Three lists of 154 word pairs presenting similar average levels of emotionality $(1.8 \pm 0.3$ vs $1.6 \pm 0.2$ vs $1.6 \pm$ $0.1 ; p>0.1)$ and word length $(7.4 \pm 2.8$ vs $7.1 \pm 2.5$ vs $7.6 \pm 2.1 ; p>0.1)$ were created. The lists differed, however, in the level of concreteness of the words composing the associates, in such a way that one list contained more abstract words than the other one $[3.6 \pm 0.3$ for one learning list (A) vs $2.8 \pm 0.3$ for the other learning list (B) vs $2.6 \pm 0.2$ for the control condition; $F_{(2,22)}=19.8 ; p<0.005$; learning list $\mathrm{A}>$ learning list $\mathrm{B}$ and learning list $\mathrm{A}>$ control list, $t$ test for paired samples, both $p$ values $<0.005]$. This difference in concreteness was confirmed in a post hoc analysis of the concreteness ratings given by the study participants at the end of each session $\left(F_{(2,24)}=16.35 ; p<0.0005 ; 3.6 \pm 0.3\right.$ for the learning list $A, 2.6 \pm 0.2$ for the learning list $\mathrm{B}$, and $2.5 \pm 0.2$ for the control condition; learning list $\mathrm{A}>$ learning list $\mathrm{B}$ and learning list $\mathrm{A}>$ control list, $t$ test for paired samples, both $p$ values $<0.005)$. This manipulation resulted in a more difficult and an easier encoding condition as indexed by the number of correctly recalled word pairs at immediate recall (see Results).

Polygraphic recordings. Eight EEG channels [F3, F4, C3, C4, P3, P4, O1, $\mathrm{O} 2$, referenced against linked mastoids $(\mathrm{A} 1+\mathrm{A} 2)]$, two electrooculograms (EOG), one submental electromyogram, and one electrocardio- 
gram (ECG) signal were recorded throughout each $24 \mathrm{~h}$ session using the Vitaport digital ambulatory sleep recorder (Vitaport-3 digital recorder; TEMEC Instruments, Kerkrade, The Netherlands). All signals were filtered at $30 \mathrm{~Hz}$ (fourth-order Bessel type anti-aliasing low-pass filter, total $24 \mathrm{~dB}$ /octave), and a time constant of $1.0 \mathrm{~s}$ was used before on-line digitization (range of $610 \mu \mathrm{V} ; 12$ bit analog-to-digital converter; $0.15 \mu \mathrm{V} / \mathrm{bit}$; sampling rate at $256 \mathrm{~Hz}$ for the EEG, EOG, and ECG). The raw signals were stored on-line on a flash memory card (Viking Components) and downloaded off-line to a personal computer hard drive. Sleep stages during scheduled naps (Table 1) and the preceding nocturnal sleep episode were visually scored on a $20 \mathrm{~s}$ epoch basis (Vitaport Paperless Sleep Scoring Software; TEMEC Instruments) according to standard criteria (Rechtschaffen and Kales, 1968).

Quantitative EEG analysis. The EEGs were subjected to spectral analysis using a fast Fourier transform (10\% cosine $4 \mathrm{~s}$ window), resulting in a $0.25 \mathrm{~Hz}$ bin resolution. EEG artifacts were detected by an automated artifact detection algorithm (Vitascore, CASA; 2000 Phy Vision, Kerkrade, The Netherlands). For final data reduction, the artifact-free $4 \mathrm{~s}$ epochs were averaged over 20 s epochs. EEG power spectra were calculated during NREM sleep in the frequency range between 0.5 and $32 \mathrm{~Hz}$.

For sleep spindle detection and analysis, the EEGs were subjected to instantaneous spectral analysis using a fast time frequency transform (FTFT) (Martens, 1992). The FTFT calculates instantaneous amplitude, frequency, and bandwidth in eight frequency bands from $0-4,4-8$, $8-12,12-16,16-18,18-22,22-24,24-28$, and $28-32 \mathrm{~Hz}$. Instantaneous bandwidth is computed from the instantaneous frequency as rectified first derivate with respect to time. Therefore, the higher the frequency variability, the higher the amplitude. Based on the $4 \mathrm{~Hz}$ range of the filters, the resolution in time of the above parameters is $0.125 \mathrm{~s}$. Over a moving template of $1 \mathrm{~s}$ duration, thresholds are applied to amplitude, frequency, and bandwidth parameters to differentiate synchronized activity from ongoing noise as well as to remove artifacts (Martens, 1999). The thresholds were determined empirically on a learning set of EEG recordings to yield the closest possible agreement with visual scores (Knoblauch et al., 2003a). Incorporating the instantaneous bandwidth helped to achieve a closer agreement compared with using only an amplitude threshold. Finally, the optimized settings from the learning set of EEG recordings were applied to the data, focusing on detected synchronized spindle activity (Knoblauch et al., 2003a). Spindles were detected from the outcome of the $8-12$ and $12-16 \mathrm{~Hz}$ frequency band, but the frequency and the bandwidth threshold for spindle detection was limited to the range of $11.25-16 \mathrm{~Hz}$. These thresholds again were determined empirically and compared with the visual score. Furthermore, a duration limit ( $\geq 0.5$ and $\leq 2 \mathrm{~s}$ ) was applied to the detected spindles. As a result, the automated spindle detection algorithm allows the investigation of the amplitude and frequency of each individual spindle at a time resolution of $0.125 \mathrm{~s}$. Using this algorithm, spindle density was computed as the number of sleep spindles per $20 \mathrm{~s}$ epoch. This technique has been successfully applied for spindle data analysis in previous studies (Knoblauch et al., 2003a,b, 2005). Spindle density values calculated during a baseline night using this algorithm were found to be similar (Knoblauch et al., 2003a) to values reported in other studies using automated (Dijk et al., 1993; Wei et al., 1999) or visual (De Gennaro et al., 2000) spindle detection techniques. Additionally, a direct comparison between the detection algorithm and visual scoring disclosed slightly higher spindle density values in the former case, because the human eye often misses superimposed sleep spindles in the presence of delta waves (Knoblauch et al., 2003a).

Statistics. The statistical packages SAS (version 6.12; SAS Institute, Cary, NC) and Statistica (2000 Statistica for Windows; StatSoft, Tulsa, OK) were used. For spectral data, ANOVA for repeated measures (rANOVA) were performed between the factors of localization (frontal, central, parietal, and occipital), side (left versus right hemisphere), and condition (easy encoding, difficult encoding, and control condition) for each frequency bin $(0.75-25 \mathrm{~Hz})$. $p$ values derived from rANOVAs were based on Huynh-Feldt's corrected degrees of freedom, but the original degrees of freedom are reported. For post hoc comparisons, paired sample $t$ tests corrected for multiple comparisons (Curran-Everett, 2000) were used. rANOVAs and $t$ tests for paired samples were also applied to sleep spindle data. $t$ tests for paired samples were used for the comparison of the memory performance between the two learning lists. $F$ values were reported when the data reached statistical significance. Post hoc $t$ test values are not reported.

The sleep EEG recording at the right occipital derivation during the nap after the easy encoding condition was defective for one subject, who was excluded from the analyses including this derivation and condition.

\section{Results}

\section{Behavioral measures}

All subjects recalled significantly fewer words after the encoding condition with more abstract words ("difficult encoding condition") than after the encoding condition with more concrete words ("easy encoding condition") (supplemental Fig. S1, available at www.jneurosci.org as supplemental material), both during the immediate recall $(48.3 \pm 5.2 \%$ for the difficult encoding vs $66.0 \pm 5.0 \%$ for the easy encoding condition; $t_{(12)}=11.4 ; p<$ $0.0001)$ and during the delayed post-nap recall $(48.7 \pm 5.1 \%$ for the difficult encoding vs $66.1 \pm 5.0 \%$ for the easy encoding condition; $\left.t_{(12)}=9.0 ; p<0.0001\right)$. Memory performance between immediate and delayed recall did not significantly change and remained stable in both encoding conditions (in both cases, $p>$ 0.75) (supplemental Fig. S1, available at www.jneurosci.org as supplemental material).

As assessed on a seven-point Likert scale, subjects rated the creation of associations between more concrete words less difficult than between more abstract words $\left(t_{(12)}=3.8\right.$; $p<0.005$; $2.8 \pm 0.2$ for the easy vs $2.1 \pm 0.2$ for the difficult condition).

Subjective ratings (five-point Likert scale) of cognitive strain, feeling of weariness, and difficulty were not different between conditions ( $p>0.8$ for all comparisons). Whereas the easy and the difficult encoding conditions did not differ in the level of boredom ( $3.1 \pm 0.3$ vs $3.1 \pm 0.3 ; p>0.05)$, the easy encoding task was rated less boring than the nonlearning control task (3.9 \pm $0.2 ; p<0.05$; interaction term, $\left.F_{(2,24)}=3.9 ; p<0.05\right)$.

\section{Circadian melatonin phase and subjective sleepiness}

Melatonin expression is an important measure because its represents the main indirect marker of the endogenous circadian rhythm in humans, and it is well known that the circadian timing system exerts its influence on cognitive performance measures, among them also memory performance (Cajochen et al., 1999; Wyatt et al., 1999; Wright et al., 2002). Because we took care to 

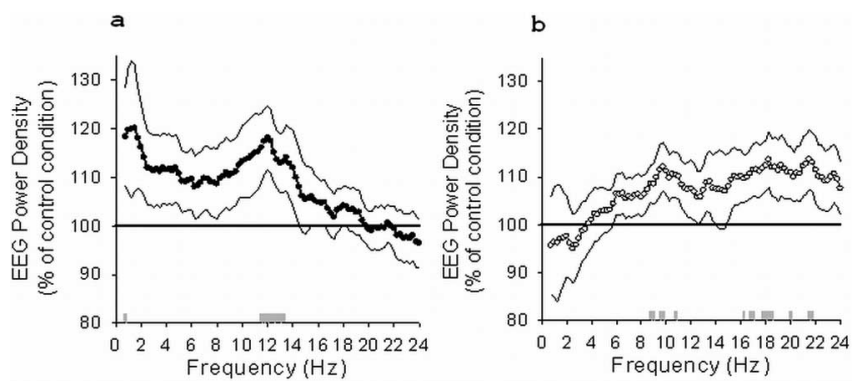

Figure 2. Relative EEG power density (percentage of control condition) during NREM sleep for F3 after the difficult encoding condition ( $\boldsymbol{a}$ ) and the easy encoding condition (b). 100\% indicates control condition, black reference line. SEMs are illustrated by dashed lines. Gray bars indicate the significant increase of EEG power density after the learning compared with the control condition (paired $t$ test comparisons).

control for both homeostatic and circadian parameters in this study, all subjects were tested at the same circadian phase, which was verified post hoc based on melatonin data. There were no significant interactions between the time course and the conditions $(p>0.75$ for KSS and VAS sleepiness scales; $p>0.2$ for salivary melatonin) (supplemental Fig. S2, available at www. jneurosci.org as supplemental material) for any of these measures, nor an effect of the condition ( $p>0.2$ for salivary melatonin; $p>0.5$ for VAS and KSS values). Post hoc estimation of circadian phase confirmed the individual's time schedule choice from actimetry data of the preceding week: the melatonin midrange crossing, an indirect marker of circadian phase, correlated positively with the sleep midpoint of actimetry data $(r=0.78 ; p<$ 0.001).

\section{Sleep variables derived from visual scoring and memory performance}

Sleep parameters during the posttraining naps (Table 1) and the night before learning did not differ between the easy and difficult encoding conditions and the nonlearning control condition (all $p$ values $>0.2$ )

\section{EEG power density during the nap and memory performance} There was no significant three-way interaction between the factors condition, derivation, and brain hemisphere (left, right) for EEG power density in any frequency bin between 0.75 and $25 \mathrm{~Hz}$ during NREMS stages 2, 3, and 4. The main factor condition yielded significance for the following frequency bins: 2, 2.5, 9.59.75, 11.5-12.75, 13.25, 17, 18, 18.25, and $18.5 \mathrm{~Hz}$ for F3; 1.5 and $2 \mathrm{~Hz}$ for $\mathrm{C} 3$; $0.75,1,1.25$, and $1.75 \mathrm{~Hz}$ for $\mathrm{O} 1$; and $1 \mathrm{~Hz}$ for $\mathrm{O} 2$ $\left(F_{(2,24)} \leq 3.7\right.$; all $p$ values $\left.<0.05\right)$.

Post hoc comparisons ( $t$ test for paired samples) showed that EEG power density after the difficult encoding condition increased significantly compared with the control condition in the left frontal derivation (F3) in the low spindle frequency range $(11.5-13.25 \mathrm{~Hz}$; all $p$ values $<0.05$ ) (Fig. $2 a$ ) (for EEG power density including only stage 2 sleep, see supplemental Fig. S3, available at www.jneurosci.org as supplemental material) and in a single frequency bin in the delta range $(0.75 \mathrm{~Hz} ; p<0.05)$ (Fig. $2 a$ ). Additionally, we detected increased EEG power density in the left central derivation (C3) in similar frequency bands but only for two single frequency bins $(2 \mathrm{~Hz}$ and $13 \mathrm{~Hz}, p<0.05)$. The comparison between the easy encoding and the nonlearning control condition revealed an increase in EEG power density for frequency bins in the alpha $(8.75-9,9.5-9.75$, and $10.75 \mathrm{~Hz}$; all $p$ values $<0.05)$ and in the beta $(16.25,16.75,17,17.75,18-18.5$,
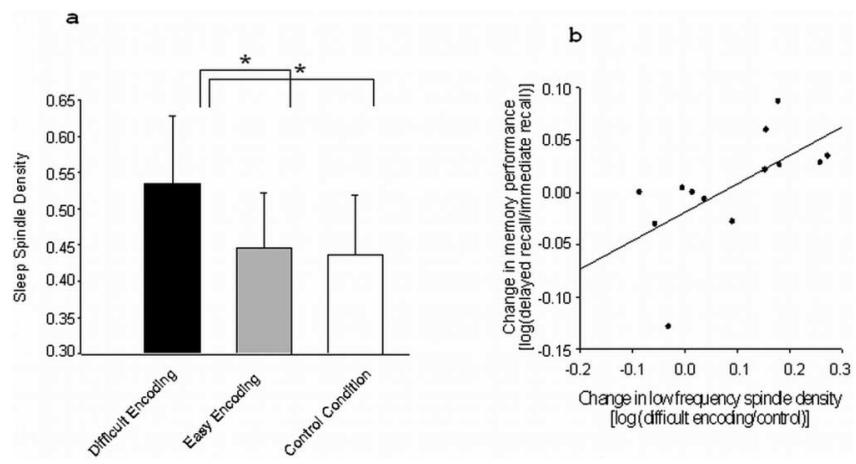

Figure 3. $\quad \boldsymbol{a}$, Low-frequency $(12.25-13.75 \mathrm{~Hz})$ sleep spindle density for $\mathrm{F} 3$ per 20 s epoch during stage 2 in the nap sleep episode. Black bar, Difficult encoding condition; gray bar, easy encoding condition; and white bar, control condition (+SEM). ${ }^{*} p<0.05 . \boldsymbol{b}$, Pearson's correlation between over-nap memory performance change [log10(delayed recall/immediate recall)] and changes in low-frequency sleep spindle density [log10(difficult encoding/control condition)].

20, 21.5-21.75, and $28.5 \mathrm{~Hz}$; all $p$ values $<0.05)$ range for the left frontal derivation (F3) (Fig. 2b). EEG power density decreased in the occipital derivation for frequency bins in the delta range $(0.75$ and $1 \mathrm{~Hz}$ for $\mathrm{O} 1, p<0.05 ; 1$ and $1.25 \mathrm{~Hz}$ for $\mathrm{O} 2, p<0.05)$ and increased for a single frequency bin in the beta range for this derivation $(22.75 \mathrm{~Hz}$ for $\mathrm{O} 2 ; p<0.05)$.

After collapsing frequency bins into broader frequency bands, we observed a significant increase in the low spindle frequency range $(11.25-13.75 \mathrm{~Hz}$ ) in F3 ( $t$ test for paired samples, $p<0.05$ ) for the difficult encoding condition and in the alpha $(8-11 \mathrm{~Hz})$ and beta $(17-20 \mathrm{~Hz})$ range for the easy encoding condition ( $t$ test for paired samples, $p<0.05)$ compared with the nonlearning control condition.

There was a positive correlation between the over-nap memory performance change (immediate vs delayed) for the difficult encoding condition and relative EEG power density (percentage of control condition) in the spindle frequency range during the post-encoding nap (for the low-frequency spindle range: C3, $r=$ $0.61, p<0.05 ; \mathrm{P} 3, r=0.60, p<0.05 ; \mathrm{O} 1, r=0.75, p<0.005$; and $\mathrm{P} 4, r=0.56, p<0.05$; for the high-frequency spindle range: $\mathrm{C} 3$, $r=0.69, p<0.01 ; \mathrm{P} 3, r=0.69, p<0.01$; and O1, $r=0.72, p<$ $0.01 ; 14-16 \mathrm{~Hz}$ ). For the easy encoding condition, results showed a positive correlation between the over-nap memory performance change and relative EEG activity in the theta range in the left frontal derivation ( $\mathrm{F} 3 ; r=0.56 ; p<0.05)$.

\section{Sleep spindle activity}

rANOVAs with the factors derivation, side, and condition were performed to compare spindle activity between the three conditions. Spindles were grouped into low-frequency spindles $(11.25-13.75 \mathrm{~Hz})$ and high-frequency spindles $(14-16 \mathrm{~Hz})$. Sleep spindle density (i.e., the number of sleep spindles per $20 \mathrm{~s}$ epoch) was determined during stage 2 sleep after the learning and nonlearning tasks. There was a main effect of the factor condition $\left(F_{(2,24)}=3.5 ; p<0.05\right)$ for the low-frequency spindles.

Post hoc analyses ( $t$ tests for paired samples) revealed an increase in low-frequency sleep spindle density in the frontocentral areas during the nap after the difficult encoding condition compared with the control condition [F3, $0.54 \pm 0.09$ vs $0.43 \pm 0.08$ (Fig. $3 a$ ); C3, $0.36 \pm 0.06$ vs $0.27 \pm 0.06$; and C4, $0.34 \pm 0.06$ vs $0.25 \pm 0.06$; all $p$ values at least $<0.05]$. No such increase was observed for the easy encoding condition compared with the control condition or for the high-frequency spindles after both 
encoding conditions. Finally, we found a positive association between over-nap memory performance change and the density change in low-frequency sleep spindles for the difficult encoding condition when compared with the control condition in $\mathrm{C} 3(r=$ $0.63 ; p<0.05$ ) (Fig. 3b). No such relationship was observed for the easy encoding condition.

\section{Discussion}

We provide here evidence that declarative learning before a daytime sleep episode leads to increased synchronized EEG oscillations during posttraining sleep, particularly in the sleep spindle range. Thus, our findings generalize the phenomenon of postlearning changes in electrophysiological activity to daytime sleep episodes. Most importantly, we could show that the nature of the declarative material to be learned and the related encoding difficulty are determinant factors for EEG changes during posttraining NREMS. After the difficult encoding condition, EEG power density increased in the low spindle frequency and in the slowwave activity range in the left frontal location. Consistently, there was a significant increase in spindle density for low-frequency spindles after the difficult encoding condition.

Our aim was to compare two memory tasks that depend on the same declarative episodic verbal memory system but differ in their encoding difficulty. This was achieved by manipulating the concreteness level of the word associates, although for both lists only, unrelated word pair associates were used. Consistent changes in posttraining sleep spindle parameters were only observed after the difficult but not after the easy associative encoding condition. This result is in agreement with animal studies, which found alterations in posttraining sleep architecture only when the training task was difficult enough (Smith and Wong, 1991). Beyond task difficulty, however, noun concreteness as well as the familiarity of the word pairs are known to affect encoding in humans (Day and Bellezza, 1983; Marschark and Surian, 1992). During the learning session, we instructed the subjects to imagine a visual relationship between the word associates of a given pair. Imagery abilities can be directly affected by the level of concreteness of the words presented and may therefore explain the observed difference of performance between the more abstract and the more concrete lists. It has been proposed that imagery is based on a general knowledge of how classes of concrete objects interact in the physical world (Day and Bellezza, 1983). In this framework, when presented with a word pair containing concrete nouns such as "peach-chair," one might more easily create a visual image of the scene, because some preexisting information is available in memory that can help to relate the two words in the present pair. When attempting to establish a visual relationship between more abstract words, such as "union-rate," one cannot as easily take advantage of specific experiences, and the resulting association is therefore intrinsically more novel. Accordingly, our participants rated the abstraction level of the association between words in a pair higher when those words were taken from the abstract wordlist. A consequential hypothesis would be that the formation of a visual relationship between words in the difficult encoding condition requires the creation of entirely new memory associations, whereas building a visual relationship in a word pair composed of concrete words would rely rather on preexisting representations that makes unrelated word pairs in the easy encoding condition akin to related word pairs.

Although it has been suggested that the creation of new associations is especially contingent on the activity of the hippocampus (Stickgold, 2004) and there is evidence that the hippocampus participates in posttraining memory consolidation for declara- tive memories (Peigneux et al., 2004, 2006; Takashima et al., 2006), the hypothesis of a prominent role of the hippocampus in the difficult encoding condition remains to be demonstrated via direct recording, e.g., using functional brain imaging studies.

Alternatively, the complementary notion of encoding depth (Craik and Lockhart, 1972) may account for differences in daytime sleep EEG oscillations after the easy and difficult learning sessions. It is known that memory encoding is influenced by both the nature of the to-be-remembered information and the way the information is processed (Guo et al., 2004). Craik and Lockhart (Craik and Lockhart, 1972; Craik, 2002) stated that “ ...good memory performance reflects deeper memory processing in the sense of more abstract semantic analysis" (Craik, 2000). In this perspective, deep encoding during the difficult condition would require a more active or elaborated processing as new memory associations need to be built. Additional experiments are needed to partial out these conjectures, which may help to reconcile result discrepancies between early (Bertini and Torre, 1973; Castaldo et al., 1974) and later (Plihal and Born, 1997; Schabus et al., 2004) studies having used similar kind of declarative learning material. Nonetheless, it is worth noticing here that we also observed changes in postlearning sleep EEG power density after the easy encoding condition. Thus, the relationship between related word pairs and different levels of abstraction in the association between unrelated word pairs should probably be best viewed in terms of a continuum rather than dichotomy.

Likewise, during nocturnal sleep (Gais et al., 2002), learningdependent EEG changes during daytime sleep were predominantly seen in the spindle frequency range after the difficult encoding condition, despite the fact that sleep spindles are under particularly pronounced circadian modulation and exhibit a striking day-night difference (Dijk and Czeisler, 1995; Dijk et al., 1997; Wei et al., 1999; Knoblauch et al., 2003a). The impact of circadian modulation varies between brain regions, but, during daytime sleep, sleep spindles are generally less abundant and have lower amplitude and shorter duration, and the within-spindle frequency is higher and more variable (Knoblauch et al., 2003a). Furthermore, we found that only low-frequency sleep spindles increased after learning in the difficult encoding condition, with largest increases in left frontal brain areas. Mean spindle frequency is lower in frontal than in centroparietal brain areas (Scheuler et al., 1990; Werth et al., 1997; Zeitlhofer et al., 1997; Anderer et al., 2001), which led some authors to propose the existence of two distinct spindle generators: frontal and parietal (Zeitlhofer et al., 1997; Anderer et al., 2001). In this perspective, our data may support the hypothesis of a specific functional implication of frontocentrally located spindles in declarative memory processes, which may be functionally dissociated from parietal-type spindles (Doran, 2003). It must be noticed, however, that frequency differences between sleep spindles recorded at different brain sites do not necessarily imply functionally distinct spindle activities. At the level of rhythmic activity in the brain, spindle and faster oscillatory activity have both been linked to aspects of memory consolidation processes (Siapas and Wilson, 1998; Steriade and Amzica, 1998; Destexhe et al., 1999; Sejnowski and Destexhe, 2000; Gais et al., 2002). Spindle oscillations provoke a massive $\mathrm{Ca}^{2+}$ entry into the spindling cortical cells and thus could set the stage for plastic synaptic changes that are supposed to be induced during subsequent slow-wave activity (Mölle et al., 2002). Furthermore, sleep spindles are temporally related to the occurrence of hippocampal ripples (Siapas and Wilson, 1998; Sirota et al., 2003), and their coactivation is be- 
lieved to be important for NREMS-dependent processes of consolidation (Buzsaki, 1998).

The widespread modifications in EEG power density during NREMS (increase in alpha and beta range at the left frontal derivation; in slow-wave activity and beta range at occipital derivations) found after the easy encoding condition are unlikely attributable to interindividual differences, because we used a repeated-measure intrasubject design. In contrast with other studies, we carefully monitored the activity of our participants during $24 \mathrm{~h}$, including the $18 \mathrm{~h}$ episode before the learning session. They were tested at the same circadian phase and under equivalent homeostatic sleep pressure in the three experimental conditions. This rigorous control ensured that posttraining sleep differences cannot be attributed to confounding effects of circadian and/or homeostatic factors, which are known to modulate performance in several memory and vigilance measures (Cajochen et al., 1999, 2004; Wyatt et al., 1999; Wright et al., 2002). Accordingly, subjective cognitive strain, difficulty, and feelings of weariness were similarly rated in the learning and control tasks. We aimed at making the control task to resemble the learning tasks as much as possible, except for the intentional learning component. Together with the homeostatic and circadian control and the fact that the two learning tasks differed only by the abstraction degree of the association between word pairs, we are reasonably sure that the observed effects genuinely represent learning-dependent changes more than a global use-dependent recovery phenomenon. This does not exclude the possibility that increased EEG spectral power in the delta range after the difficult encoding condition reflects local posttraining recovery in the areas solicited by the encoding effort. Finally, we observed that increased activity in EEG power spectra in the low spindle frequency range and spindle density was positively correlated with changes in memory performance between pre-sleep and postsleep recall sessions. This result further suggests that spindle activity during daytime sleep is involved in the consolidation of recently acquired declarative memories in humans, at least in the difficult encoding condition. Hence, our results are in agreement with nocturnal sleep studies using declarative material (Plihal and Born, 1997; Gais et al., 2002; Gais and Born, 2004; Schabus et al., 2004; Clemens et al., 2005) (for spatial episodic memory, see Peigneux et al., 2004) and extend those findings in showing that learning-related changes in sleep parameters and sleep-related consolidation of declarative memories are contingent on the nature of the material to be learned.

\section{References}

Akerstedt T, Gillberg M (1990) Subjective and objective sleepiness in the active individual. Int J Neurosci 52:29-37.

Anderer P, Klosch G, Gruber G, Trenker E, Pascual-Marqui RD, Zeitlhofer J, Barbanjoj MJ, Rappelsberger P, Saletu B (2001) Low-resolution brain electromagnetic tomography revealed simultaneously active frontal and parietal sleep spindle sources in the human cortex. Neuroscience 103:581-592.

Bertini M, Torre A (1973) REM sleep and memory consolidation. In: Sleep: physiology, biochemistry, psychology, pharmacology, clinical implications (Levin P, Koella WP, eds), pp 453-457. Basel: Karger.

Buzsaki G (1998) Memory consolidation during sleep: a neurophysiological perspective. J Sleep Res 7 [Suppl 1]:17-23.

Cajochen C, Khalsa SB, Wyatt JK, Czeisler CA, Dijk DJ (1999) EEG and ocular correlates of circadian melatonin phase and human performance decrements during sleep loss. Am J Physiol 277:R640-R649.

Cajochen C, Knoblauch V, Wirz-Justice A, Krauchi K, Graw P, Wallach D (2004) Circadian modulation of sequence learning under high and low sleep pressure conditions. Behav Brain Res 151:167-176.

Castaldo V, Krynicki V, Goldstein J (1974) Sleep stages and verbal memory. Percept Mot Skills 39:1023-1030.
Chrobak JJ, Buzsaki G (1996) High-frequency oscillations in the output networks of the hippocampal-entorhinal axis of the freely behaving rat. J Neurosci 16:3056-3066.

Clemens Z, Fabo D, Halasz P (2005) Overnight verbal memory retention correlates with the number of sleep spindles. Neuroscience 132:529-535.

Craik FIM (2002) Levels of processing: past, present, and future? Memory 10:305-318.

Craik FIM, Lockhart RS (1972) Levels of processing: a framework for memory research. J Verbal Learn Verbal Behav 11:671-684.

Curran-Everett D (2000) Multiple comparisons: philosophies and illustrations. Am J Physiol Regul Integr Comp Physiol 279:R1-R8.

Day JC, Bellezza FS (1983) The relation between visual imagery mediators and recall. Mem Cognit 11:251-257.

Destexhe A, Contreras D, Steriade M (1999) Cortically-induced coherence of a thalamic-generated oscillation. Neuroscience 92:427-443.

Dijk DJ, Czeisler CA (1995) Contribution of the circadian pacemaker and the sleep homeostat to sleep propensity, sleep structure, electroencephalographic slow waves, and sleep spindle activity in humans. J Neurosci 15:3526-3538.

Dijk DJ, Hayes B, Czeisler CA (1993) Dynamics of electroencephalographic sleep spindles and slow wave activity in men: effect of sleep deprivation. Brain Res 626:190-199.

Dijk DJ, Shanahan TL, Duffy JF, Ronda JM, Czeisler CA (1997) Variation of electroencephalographic activity during non-rapid eye movement and rapid eye movement sleep with phase of circadian melatonin rhythm in humans. J Physiol (Lond) 505:851-858.

Doran SM (2003) The dynamic topography of individual sleep spindles. Sleep Res Online 5:133-139.

Gais S, Born J (2004) Low acetylcholine during slow-wave sleep is critical for declarative memory consolidation. Proc Natl Acad Sci USA 101:2140-2144.

Gais S, Molle M, Helms K, Born J (2002) Learning-dependent increases in sleep spindle density. J Neurosci 22:6830-6834.

Guo C, Zhu Y, Ding J, Fan S, Paller KA (2004) An electrophysiological investigation of memory encoding, depth of processing, and word frequency in humans. Neurosci Lett 356:79-82.

Hager W, Hasselhorn M (1994) Handbuch deutschsprachiger Wortnormen. Göttingen, Germany: Hogrefe, Verlag für Psychologie.

Hofer-Tinguely G, Achermann P, Landolt HP, Regel SJ, Retey JV, Durr R, Borbely AA, Gottselig JM (2005) Sleep inertia: performance changes after sleep, rest and active waking. Brain Res Cogn Brain Res 22:323-331.

Huber R, Ghilardi MF, Massimini M, Tononi G (2004) Local sleep and learning. Nature 430:78-81.

Jewett ME, Wyatt JK, Ritz-De Cecco A, Khalsa SB, Dijk DJ, Czeisler CA (1999) Time course of sleep inertia dissipation in human performance and alertness. J Sleep Res 8:1-8.

Knoblauch V, Martens WLJ, Wirz-Justice A, Kräuchi K, Cajochen C (2003a) Regional differences in the circadian modulation of human sleep spindle characteristics. Eur J Neurosci 18:155-163.

Knoblauch V, Martens WLJ, Wirz-Justice A, Cajochen C (2003b) Human sleep spindle characteristics after sleep deprivation. Clin Neurophysiol 114:2258-2267.

Knoblauch V, Münch M, Blatter K, Martens WLJ, Schroder C, Schnitzler C, Wirz-Justice A, Cajochen C (2005) Age-related changes in the circadian modulation of sleep spindle frequency during nap sleep. Sleep 28:1093-1101.

Maquet P (2001) The role of sleep in learning and memory. Science 294:1048-1052.

Maquet P, Laureys S, Peigneux P, Fuchs S, Petiau C, Phillips C, Aerts J, Del Fiore G, Degueldre C, Meulemans T, Luxen A, Franck G, Van Der Linden M, Smith C, Cleeremans A (2000) Experience-dependent changes in cerebral activation during human REM sleep. Nat Neurosci 3:831-836.

Marschark M, Surian L (1992) Concreteness effects in free recall: the roles of imaginal and relational processing. Mem Cognit 20:612-620.

Martens WLJ (1992) The fast time frequency transform (F.T.F.T.): a novel on-line approach to the instantaneous spectrum. Paper presented at 14 th International Conference of the IEEE Engineering in Medicine and Biology Society, Paris, October.

Martens WLJ (1999) Segmentation of "rhythmic" and "noisy" components of sleep EEG, heart rate and respiratory signals based on instantaneous amplitude, frequency, bandwidth and phase. Paper presented at 1st Joint BMES/EMBS IEEE Conference, Atlanta, 1999. 
Meier-Koll A, Bussmann B, Schmidt C, Neuschwander D (1999) Walking through a maze alters the architecture of sleep. Percept Mot Skills 88:1141-1159.

Mölle M, Marshall L, Gais S, Born J (2002) Grouping of spindle activity during slow oscillations in human non-rapid eye movement sleep. J Neurosci 22:10941-10947.

Peigneux P, Laureys S, Fuchs S, Destrebecqz A, Collette F, Delbeuck X, Phillips C, Aerts J, Del Fiore G, Degueldre C, Luxen A, Cleeremans A, Maquet P (2003) Learned material content and acquisition level modulate cerebral reactivation during posttraining rapid-eye-movements sleep. NeuroImage 20:125-134.

Peigneux P, Laureys S, Fuchs S, Collette F, Perrin F, Reggers J, Phillips C, Del Fiore G, Aerts J, Luxen A, Maquet P (2004) Are spatial memories strengthened in the human hippocampus during slow wave sleep? Neuron 44:535-545.

Peigneux P, Orban P, Balteau E, Degueldre C, Luxen A, Laureys S, Maquet P (2006) Offline persistence of memory-related cerebral activity during active wakefulness. PLoS Biol 4:e100.

Plihal W, Born J (1997) Effects of early and late nocturnal sleep on declarative and procedural memory. J Cogn Neurosci 9:534-547.

Rauchs G, Desgranges B, Foret J, Eustache JF (2005) The relationships between memory systems and sleep stages. J Sleep Res 14:123-140.

Rechtschaffen A, Kales A (1968) A manual of standardized terminology, techniques and scoring system of sleep stages of human subjects. Bethesda, MD: United States Department of Health, Education, and Welfare, Public Health Service.

Schabus M, Gruber G, Parapatics S, Sauter C, Klosch G, Anderer P, Klimesch W, Saletu B, Zeitlhofer J (2004) Sleep spindles and their significance for declarative memory consolidation. Sleep 27:1479-1485.

Schabus M, Hödlmoser K, Pecherstorfer T, Klösch G (2005) Influence of midday naps on declarative memory performance and motivation. Somnologie 9:148-153.

Scheuler W, Kubicki S, Scholz G, Marquardt J (1990) Two different activities in the sleep spindle frequency band-discrimination based on the topographical distribution of spectral power and coherence. In: Sleep '90 (Horne J, ed), pp 13-16. Bochum, Germany: Pontenagel.

Sejnowski TJ, Destexhe A (2000) Why do we sleep? Brain Res 886:208-223.

Siapas AG, Wilson MA (1998) Coordinated interactions between hippocampal ripples and cortical spindles during slow-wave sleep. Neuron 21:1123-1128.

Sirota A, Csicsvari J, Buhl D, Buzsaki G (2003) Communication between neocortex and hippocampus during sleep in rodents. Proc Natl Acad Sci USA 100:2065-2069.
Smith C (1995) Sleep states and memory processes. Behav Brain Res 69:137-145

Smith C (2001) Sleep states and memory processes in humans: procedural versus declarative memory systems. Sleep Med Rev 5:491-506.

Smith C, Wong PT (1991) Paradoxical sleep increases predict successful learning in a complex operant task. Behav Neurosci 105:282-288.

Steriade M, Amzica F (1998) Coalescence of sleep rhythms and their chronology in corticothalamic networks. Sleep Res Online 1:1-10.

Stickgold R (2004) Dissecting sleep-dependent learning and memory consolidation. Comment on Schabus $\mathrm{M}$ et al., Sleep spindles and their significance for declarative memory consolidation (Sleep 27:1479-1485). Sleep 27:1443-1445.

Stickgold R, Walker MP (2005) Sleep and memory: the ongoing debate. Sleep 28:1225-1227.

Takashima A, Petersson KM, Rutters F, Tendolkar I, Jensen O, Zwarts MJ, McNaughton BL, Fernandez G (2006) Declarative memory consolidation in humans: a prospective functional magnetic resonance imaging study. Proc Natl Acad Sci USA 103:756-761.

Torsvall L, Åkerstedt T (1980) A diurnal type scale. Construction, consistency and validation in shift work. Scand J Work Environ Health 6:283-290.

Walker MP, Stickgold R (2006) Sleep, memory, and plasticity. Annu Rev Psychol 57:139-166

Weber JM, Schwander JC, Unger I, Meier D (1997) A direct ultrasensitive RIA for the determination of melatonin in human saliva: comparison with serum levels. J Sleep Res 26:757.

Wei HG, Riel E, Czeisler CA, Dijk DJ (1999) Attenuated amplitude of circadian and sleep-dependent modulation of electroencephalographic sleep spindle characteristics in elderly human subjects. Neurosci Let 260:29-32.

Werth E, Achermann P, Dijk DJ, Borbely AA (1997) Spindle frequency activity in the sleep EEG: individual differences and topographic distribution. Electroencephalogr Clin Neurophysiol 103:535-542.

Wright KP, Hull JT, Czeisler CA (2002) Relationship between alertness, performance, and body temperature in humans. Am J Physiol Regul Integr Comp Physiol 283:R1370-R1377.

Wyatt JK, Ritz-De Cecco A, Czeisler CA, Dijk DJ (1999) Circadian temperature and melatonin rhythms, sleep, and neurobehavioral function in humans living on a 20-h day. Am J Physiol 277:R1152-R1163.

Zeitlhofer J, Gruber G, Anderer P, Asenbaum S, Schimicek, Saletu B (1997) Topographic distribution of sleep spindles in young healthy subjects. Sleep Res 6:149-155. 